\title{
Research of Electric Motor Multi-type Soft Start Control Mode Based on over-load Protection
}

\author{
Lina Liu \\ School of Electric and Electronic Engineering, Shandong University of Technology,Zibo, China, \\ Email:linaliu-126@163.com \\ Jishun Jiang and Liang Zhang \\ School of Electric and Electronic Engineering, Shandong University of Technology,Zibo, China, \\ Email: jandj8@sina.com, zhangdaozheng333@126.com
}

\begin{abstract}
This article in view of the question that the high power motor could not starting and stopping directly in the industry, proposed one kind of design based on PIC single chip high power motor soft start and intelligence protective devices, utilized real-time measurement and control method with the feature of synchronous sampling electric current spurt value inverse time lag protection, according to the load situation four starting and protection control soft start ways of the under intelligent protection controller were designed. Intelligent soft starter design principles based on PIC single chip is articulated. The hardware circuit design, software flow design and test data analysis are given in details. By producing in Zibo Galaxy high-technology development co., Ltd., it shows that this smart soft starter has the characteristics which are flexible parameter setting, intuitive liquid crystal display, diverse starting way, precise current limiting protection, accurate protection of lack phase, low-cost, collecting soft start and running protection with a body's protection controller, are suitable specially for the starting and control of high power motor, so the protector has broad application prospects.
\end{abstract}

Index Terms-motor, soft start, over-load protection, PIC single chip

\section{INTRODUCTION}

The high power three-phase asynchronous motor is widely applied in each kind of industrial field by its structure simply and perform reliably. The high power motor will produce a very big surge current to the electrical network in the direct starting instance, the mechanical drive part will have the breakdown as a result of the impact torque. Moreover, the direct starting can burn out the winding easily, does not permit direct starting with load. Therefore, people also unceasingly improve the starting technology of three-phase asynchronous motor. The soft start technology develops from initial stator series resistance, $\mathrm{Y}-\Delta$ starting, autotransformer pressure reduction starting to the intelligent soft start that is controlled by modern thyristor pressure regulating. The traditional soft start control system has many disadvantages which are the structure is complex, the part is numerous and the volume is huge, which causes the failure rate to be high, service difficulty and maintenance cost higher[1-3]. A novel design of intelligent soft starter based on 89LV52 single chip is proposed in this article, the design uses PWM regulator module voltage starting chopping control, controls the completion of the internal bypass relay to achieve bypass control after starting, adpots current sensor for motor current measurement, uses phase lacking detection circuit for three-phase motor to realize phase detection, system has a flexible key set, intuitive LCD, starting way is diverse, current limiting protection precisely; lack of phase protection accurately; it has the characteristics which are structure simple, low cost, design concepts innovative, and so on.

\section{WORKING PRINCIPLE OF SOFT START CONTROLLER}

\section{A. Working priciple of soft startor [4-6]}

Smart soft starter adopts three opposite parallel thyristor MJSY-QKJL module as a regulator, connects it between the power supply and stator of the motor. Its basic principle is to use principle of phase shift and control of SCR, based on a predetermined different way the control circuit starts the synchronization through detection voltage signals as benchmarks of phase shift control pulse, and detects the feedback current signal of primary circuit, compares with the setting value to calculate the volume control for adjusting the control voltage of MJSY-QKJL module, thus realizes the angle $\alpha$ control of SCR and AC output voltage adjustment, so that the side voltage of motor gradually increases at a set speed, to achieve the aim of controlling starting current and torque by voltage adjusting methods. The relationship of DC controls input voltage Vin and AC output voltage Vout of thyristor control module MJSY-QKJL as shown in table 1.

TABEL1 THE RELATIONSHIP OF DC CONTROLS INPUT VOLTAGE Vin and AC OUTPUT VOLTAGE Vout OF THYRISTOR MJSY-QKJL MODUL CONTROLS

\begin{tabular}{ccccccc}
\hline index & 1 & 2 & 3 & 4 & 5 & 6 \\
\hline Input voltage(V) & 0.0 & 1.0 & 1.5 & 2.0 & 2.5 & 3.0 \\
Output voltage(V) & 0 & 2 & 14 & 40 & 76 & 123 \\
\hline index & 7 & 8 & 9 & 10 & 11 & 12 \\
\hline Input voltage(V) & 3.5 & 4.0 & 4.5 & 5.0 & 6.0 & 7.0 \\
Output voltage(V) & 186 & 245 & 296 & 334 & 375 & 380 \\
\hline
\end{tabular}

The relationship of module DC input voltage and 
AC output voltage is nonlinear relations, piecewise linear approach can be used to calculate the relationship between inputs and outputs precisely. When the motor completes starting and reaches rated voltage, the motor can directly connect to the power system for running through isolator controls the closing of the three-phase bypass relay. Due to the soft starter is electronic voltage regulator and detecting the current, therefore also has the functions that heat protection on the motor and soft starter, limiting the impacts of torque and current, protections of three-phase power imbalances, phase lacking and phase breaking, and so on. After overload protection tripping, the soft start again breaks connection in a short time, the module gives off heat seriously, requires certain radiation time, establish certain restoration time to guarantee the soft start could not start in the restore time, normally the time interval is settled at 3 5 minutes.

\section{B. Starting modes of soft startor}

There are four soft starting modes that are current limiting starting, voltage ramp starting, voltage ramp and current restriction soft starting, kick torque starting. According to different load and specific use conditions the user can select different starting mode to achieve optimization combination of starting current and torque.

(a) The current limiting soft start control mode uses the electric current negative feedback closed-loop control, the given value is the limited current value, uses current transformer detect the actual value of armature current, the deviation of them is used by PID regulator to calculate the value of exports $U(\mathrm{k})$, then the control voltage of the MJSY-QKJL module is adjusted by $U(\mathrm{k})$, therefore, adjusting initial starting not surpass the user setting value is realized by the electric current loop. When starting, the electric current rise to the setting value by certain slope, after that the maintenance is constant until the starting finished. Then the bypass relay puts into work, the thyristor MJSY-QKJL module stops working. The restriction value of electric current may be adjusted according to the net load situation, the range of set value is 1 to 5 times of the motor rating current Ie. In this mode, the starting current is small way, according to demand the adjustment can be marked, has small influence to the electrical network, adapts in overwhelming majority application situation.

(b) The mathematical model of voltage ramp soft starting control mode as equation (1),

$$
\mathrm{Ua}=(\mathrm{Ue}-\mathrm{Ur}) * \mathrm{t} / \mathrm{Tr}+\mathrm{Ur}
$$

In equation(1), Ua denotes the output voltage of voltage ramp soft starting pattern, Ue denotes the rated phase voltage, Ur is the initial voltage of soft starter output, Tr denotes the soft starting time of the soft start controller, $t$ denotes the current time in the soft start time. Then according to the setting soft start time t elevates the output voltage smoothly until the rated voltage Ue is obtained along with elevation of output voltage, the rotational speed of electric motor increases unceasingly until achieves the rated speed, finishes until the starting end. Then the bypass relay puts into work, the thyristor
MJSY-QKJL module withdraws work.

(c) The voltage ramp and current restriction soft starting control mode concentrates the merits of the first two kind of starting pattern, the output voltage roses steadily according to the setting starting time, simultaneously the output current increases by certain speed, when the initial starting current increases to electric current restriction value Im, the electric current maintains constant, finishes until the starting end. Then the bypass relay puts into the work, the thyristor MJSY-QKJL module withdraws work.

(d) In kick torque soft starting control mode, Tk expresses the time on which exerts the kick initial voltage Uk, generally the set value is 3 seconds, the initial voltage is $\mathrm{Uk}=300 \mathrm{~V}$, when $\mathrm{t} \leq \mathrm{Tk}$, the soft start output voltage is $\mathrm{Ua}=\mathrm{Uk}$. when $\mathrm{t}>\mathrm{Tk}$, the $\mathrm{Ua}$ is shown in equation (2), as follows

$$
\mathrm{Ua}=(\mathrm{Ue}-\mathrm{Ur}) *(\mathrm{t}-\mathrm{Tk}) /(\mathrm{Tr}-\mathrm{Tk})+\mathrm{Ur}
$$

Where $\mathrm{Ua}$ is the soft start output voltage, Ue denotes the rated voltage, Ur is the initial voltage, Tr is the soft start time, Tk is the kicking time, $\mathrm{t}$ is the current time; Kicks the torque soft start pattern is mainly applied on the load motor which static resistance is quite big, through exerts an instant big starting torque to overcome the big static friction. Under this pattern the output voltage achieves the set kick voltage (Uk) rapidly, when reaches the set kicks time kicks time (Tk), the voltage reduces to the initial voltage, according to the set starting time elevates the output voltage smoothly again until the rated voltage $\mathrm{Ue}$ is reached, finished until the starting end. Then the bypass relay puts into work, the thyristor MJSY-QKJL module withdraws work.

\section{Stoping modes of soft startor}

There are two stop modes of soft starter, one is voltage ramp stopping, the other is emergency stopping.

The voltage ramp soft stop mode is suitable in the situation that electrical machinery load requests steady slow parking, first puts through the thyristor MJSY-QKJL module, entire breakover 0.1 second later, separates the bypass relay, then the breakover angle of the thyristor MJSY-QKJL module reduces gradually, the running voltage reduces gradually, the mathematical model of voltage ramp soft stop mode shows in equation (3), as follows

$$
\mathrm{Ua}=\mathrm{Ue}^{*}(\mathrm{Ts}-\mathrm{t}) / \mathrm{Ts}
$$

$\mathrm{Ua}$ is the output voltage of soft stop, Ue denotes the rated voltage, Ts is the soft start time, $\mathrm{t}$ is the current time; Completes the electrical machinery to stop steadily by realizing relay separation on zero voltage.

The emergency stop mode is suitable in the situation of power failure suddenly and emergency stop, first puts through the thyristor MJSY-QKJL module, entire breakover 0.02 second later, separates the bypass relay, then shutdown thyristor MJSY-QKJL module quickly, lets the electrical machinery stop fast, realizes relay separation on zero voltage, therefore the situation in which the electric spark maybe produce while the relay is separated instantaneously has been eliminated, thus the relay life is lengthened. 


\section{Principle of curren measurement}

The measurement of three-phase current effective value Ia, Ib, Ic uses LF398 retainer to carry on synchronous sampling to A, B, C three-phase alternating current, controls the LF398 retainer using switch quantity to realize the sampling synchronous maintains, the sampling period is set as 0.5 millisecond, obtains electric current spurt value Uai(k), Ubi(k), Uci(k) separately, through multi-way switch cut each sampling period $\tau$ synchronous gathering spurt value of three-phase current, each alternating current cyclical $\mathrm{T}$ surveys 40 groups of sampling points, then through the equation (2) calculates the armature current.

Calculation formula of current effective value is

$$
I=\sqrt{\frac{1}{T} \int_{0}^{T} u_{i}^{2}(t) d t}
$$

After discretized the equation is

$$
I=\sqrt{\frac{1}{n} \sum_{k=0}^{n-1} u_{i}(k \tau)}
$$

Where $\mathrm{Ui}$ is electric current spurt value, $\mathrm{T}$ is the period of AC current, $\tau$ is the sampling period, $\mathrm{n}$ is the number of sampling points. I is th effective value of AC current.

\section{THE DESIGH OF SOFT START CONTROLLER}

\section{A. Structure of the system}

Integrated control system is based on the PIC16F916 single chip, PIC16F916 chip has a 28-pin, a highly stable $8 \mathrm{MHz}$ internal oscillator, $14 \mathrm{~KB}$ Flash program memory and up to 352 bytes RAM and 256 bytes digital EEPROM, chips with under-voltage reset functions, enhanced watchdog timer (WDT), programmable low voltage detection, 3 timers, and 2 capture/compare/PWM modules, the external circuit is simple. PIN RA0-RA7,RB0-RB7,RC0-RC7 may as a general I/O and LCD analog output. I/O port number is 24. External interface circuit using the $\mathrm{I} 2 \mathrm{C}$ bus structure, is a cost-effective 8-bit single chip that is more suitable to switch quantity input signal detection. The structure of system as shown in Fig.1.

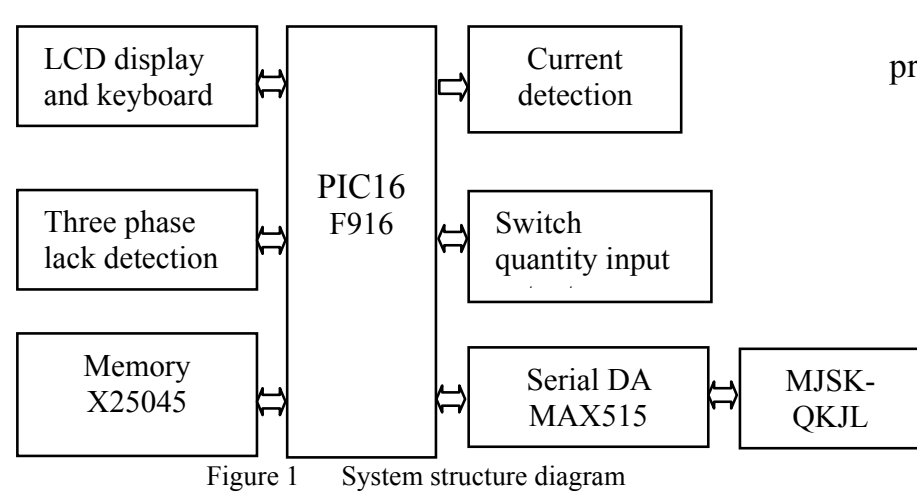

The Integrated controller hardware design mainly includes six big modules, the three-phase lacking examination electric circuit module, the LCD liquid crystal display electric circuit and the keyboard sweep circuit module, serial storage and watchdog X25045 electric circuit module, bypass relay control and switch quantity input output circuit module, three-phase current examination electric circuit module, serial DA output controls thyristor MJSY-QKJL module. The working principle of soft start controller, through the pressed key sets the ratio of current transformer, the starting way, the starting voltage, the initial starting current, the starting time, and so on stand-by time, and records them in $\mathrm{X} 25045$. Then select the start button, go to the set starting state, with the increasing of the output voltage the motor speeds up to the rated speed, after the starting end the bypass relay puts into work, thyristor MJSY-QKJL module exits. When the motor working is finished, select the stop button, enter the set soft stopping state, thyristor modules MJSY-QKJL breakover rapidly, disconnect the bypass relay, through serial DA controls thyristors MJSY-QKJL module to implement the declining of motor voltage, speeds down until the speed drops to zero, soft stop finishes. The soft start controller has the functions of flexible key setting, intuitive liquid crystal display, diverse starting way, current limiting protection and lack of protection, etc.

\section{B. LCD display and Key-press}

LCD Display:

The LCD display adopts HS12864-15B LCD display driver chip of HOLTEK to achieve 4 lines of 16 characters per line LCD digital display and Chinese character prompt. HS12864-15B is a built in Chinese character library memory LCD driver, which includes control and timing circuits, display RAM, LCD driving and bias, watchdog timer, etc.

The characteristics of HS12864-15 series Chinese graph liquid crystal display module are mainly decided by its controller ST7920. At the same time ST7920 takes the controller and driver, it may provides 33 group com outputs and 64 group seg outputs. Under the driver ST921 coordination, may actuate $256 \times 32$ lattice liquid crystal mostly.

HS12864-15 serial products of the Chinese rises industry limited company are HS12864-15 (to have V3.0 and V4.0 edition circulation market),HS12864-15B and HS12864-15C.

The hardware characteristics of HS12864-15 serial products as follows:

- provides 8,4 bit parallel connection and serial interface may choose

- parallel interface adaptive M6800 succession

- automatic power source to start reset function

- construct vibration source internal

- 64×16 bits character display RAM (DDRAM to be most 16 character $\times 4$ lines, LCD demonstrated that scope $16 \times 2$ lines)

$2 \mathrm{M}$ bits Chinese font ROM (CGROM), altogether provide 8192 Chinese fonts $(16 \times 16$ lattice)

- 16K bits half-width font (HCGROM), 
altogether provide 126 English fonts $(16 \times 8$ lattice)

- $64 \times 16$ bits character generation RAM (CGRAM)

The software characteristics of HS12864-15 serial products as follows:

writing and graph mix demonstration function
picture elimination function
cursor homing function
display open/close function
cursor demonstration/hideaway function
demonstration typeface twinkle function
cursor shifting function
demonstration shifting function
vertical picture revolving function
fiber demonstration function
dormancy pattern

It uses serial interface between PIC16F946 and itself, just three wires. RB4, RB5, RB6 and RB7 of PIC16F946 connects to foure pin respectively to control the Refresh display RAM buffer. As shown in Fig.2.

Key-press Control:

The controller has a total of eight key-press, unlock, set, increase, decrease, confirmation, start, stop and emergency stop. The key-press function divides into three big functions: parameter establishment function, start or stop function, protection and unlocking function.

The establishment function, under the stopped state, simultaneously presses down "establishment" key and "increases", enters sensor parameter establishment and whether have external connection pressed key control establishment. Under the stopped state, simultaneously presses down "the establishment" key and "reduction", enters the soft start way and parameter establishment; This time screen prompt: the current state is establishment state, and the established parameter twinkle, establishes the parameters in turn which are starting way, starting time, starting voltage, soft stop time, nominal current, current limiting electric current and so on, press once again the key to confirm, then completes soft start way and parameter establishment, enters the stopping state.

Start or stop function, at this time the screen demonstration is stopped state, current state: stop, starting way: pitch, starting time: 10 seconds. Soft stop time: 20 seconds, nominal current: 300 A, current limiting electric current: $500 \mathrm{~A}$, whether there is outside connection pressed key control: does not have. Press the "starting" the key, the motor enters soft starting condition, liquid crystal display size of initial firing current and starting time, when the starting time reduces gradually until 0 seconds, bypass relay closed, completes the start-up procedure, the motor enters rated voltage active status, and enters the overflow protection monitor state, this time liquid crystal display the motor's actual movement armature current. Press "stop" key, motor enters soft stopping state, first puts through the control thyristor MJSY-QKJL module, then separates bypass relay, according to the set soft stop time drops the voltage gradually until stop. Presses down emergency stop key, enters fast emergency stopping status, soft start stop emergently in 2 to 4 seconds. In starting and stopping state, if has external connection key-press control, through external connection key-press control motor's soft start and soft stop, then the "starting" and "stop" key does not have an effect, but emergency stop still has an effect.

Protection and unlocking, when the electrical machinery presents breakdown, motor under running status enters emergency stop condition, after stopping the system presents the following prompt: current condition: malfunction; breakdown 1: The system failure instructed; breakdown 2: phase lack protection instruction; breakdown 3: overflow protection instruction. Presents any kind of breakdown, the electric motor is at the stopped state, cannot start, and instructs the breakdown type, and is at the protection status, waited for engineers fix the current breakdowns. After trouble shooting, presses down the solution latch key, the failure indication automatic withdrawal, enters the parking instruction condition. As shown in Fig. 2.

\section{RS485 Communication}

MAX487 chip can implement RS485 communication control of integrated controller. MAX487 chip has RS485 communication protocol. It can take 128 hypogenous computers. Its transmission distance is greater than $1 \mathrm{~km}$ and its transfer rate is up to $250 \mathrm{~kb}$ per second. The integrated controller connects with the unit controller through the RS485 bus. That is to say, whose address is called, the watt-hour meter package the information and checkout code and then upload to the power management computer. DE of MAX487 is the transmitter power. The transmitter works when DE is1. DI is input and A, B is outputs. When DE is 0 , stop sending and the output is high resistance. $\mathrm{RE}$ is the input power. The receiver works when RE is 0 . A and $B$ are inputs and RO is output. When RE is 1 , the receiver is prohibited and $\mathrm{RO}$ is the high resistance state. Therefore, we adopt Half-Duplex communication and connect DE and RE to RC5 of PIC16F916 through which to control the transceiver working condition.

\section{Curremt Detection and Overflow Protection}

The three-phase current of electric motor after Hall electric current sensor examination, passes through TL084 emitter follow output, Uai, Ubi and Uci connects to three signal input ends of PIC16F916 separately, the three input ends RA0, RA1 and RA2, take advantage of the tablet in triple 10-bit A/D conversion, current data acquisition is realized, detecting three-phase current of high power motors is completed.

After the motor starts, uses the bypass relay connect the motor's main circuit, regarding the motor electric current, according to continuously overloads 1.5 time of nominal current protection movements in 5 minutes, overloads continuously 2 time of nominal current protection movements in 3 minutes, overloads continuously 3 time of nominal current protection movement in 1 minute, overloads continuously 4 time of 
nominal current protection movements in 30 seconds, overloads continuously 5 time of nominal current protection movements in 10 seconds, to 6 time of nominal current protection movements in 5 seconds. During the design to the following several points should be pay attention to:

(1) After the electric motor soft start had ended enters the fixed active status, analyzes whether to overload, the overload protection moves, thus electric motor overload protection characteristic only shunts the characteristic of the motor initial firing current, can guarantee its normal work; But its operating time cannot be too long, its characteristic can only be able to play overload protection role under the motor thermal characteristics.

(2) Overload protection instant working current should be slightly bigger than the motor start surge current. Such as, some protective device belts overload instant holding function, then its working current should be big somewhat compared to initial firing current peak value, be able to cause the motor normal starting, therefore when the motor uses kick starting, allows 4 to 5 times of the nominal current to overload does not surpass 3 seconds to kick starting.

(3) Operating time of overload protection should be small spot compared to the wire thermal characteristics, be able to have supply line reserve protection function. The overload protection and short circuits protective device sychromesh for general overload protection not to have the breaking short circuit current ability. Once has a short circuit in the movement, needs short circuit protective device which series in main circuit cut off the circuit. As shown in Fig.2.

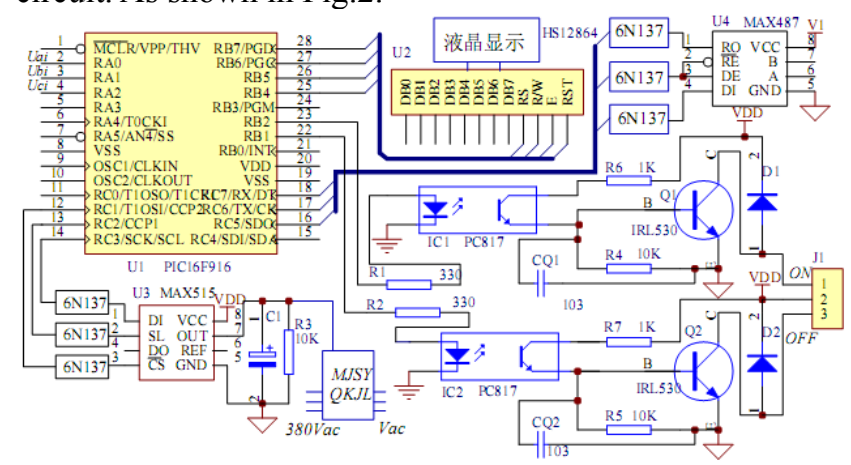

Figure 2 Part of the circuit diagram of soft startor

\section{E. Control of Thyristor MJSY-QKJL Module}

Thyristor MJSY-QKJL module is a DC voltage controlled three opposite parallel pressure regulating and control module produced by Zibo Galaxy high-technology development co., Ltd., the relationship of DC control voltage Vin and AC output voltage Vout as shown in table 1. Using the serial output of serial DA converter MAX515, through zooming and filtering the DA output connects to the control voltage Vin of MJSY-QKJL module, and adjustment of the output voltage Vout is achieved. The resolution of serial D/A converters MAX515 is 10 bit, settling time of voltage output is $20 \mathrm{~ms}$, supply voltage Vcc is $5 \mathrm{~V}$. DI denotes serial data input, SL is the serial shift pulse input, CS the chip selecting input, low level valid. The wiring is shown in Fig.2.

\section{F. Phase Lack Detection and Input Ouput of Switch Quantity}

Phase detection adopts three-phase voltage Va, Vc, $\mathrm{Vb}$, respectively and neutral V0, leads signals respectively through $75 \mathrm{~K}$ resistance and diode half-wave rectifier and capacitance filtering accesses to the optical isolator, the wiring diagram is shown in Fig.3, passes the isolator EL817, three-phase voltage switch quantity accesses RA3, RA4, RA5 pin of PIC16F916. Switch quantity processing adopts isolator, high-speed 6N137 and common EL817 optical isolator is utilized in the system. Data acquisition and output using high-speed optocoupler $6 \mathrm{~N} 137$ isolation. Input and output of the switch quantity using EL817 isolation, isolator controls the solid state relays directly, completes bypass relay control and sound-light alarming control. Using isolator by optical coupling signal transmission, the system anti-interference ability is improved.

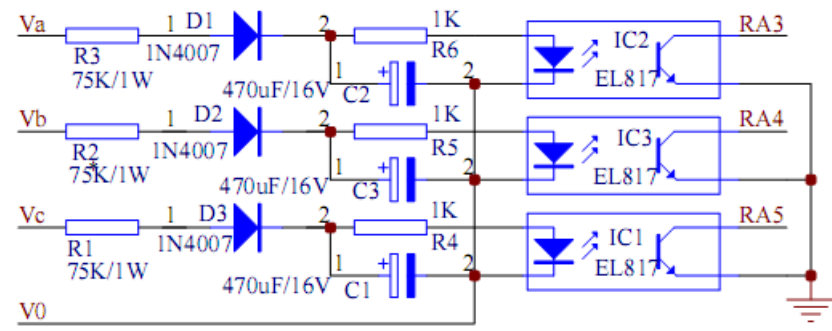

Figure 3 priciple diagram of phase lack detection

\section{THE DESIGH OF SOFTWARE PROGRAM}

\section{A. Resource Allocation of Software Program of the System}

The soft start controller uses the PIC16f916 single chip circuit to carry on control. Software program of soft start controller mainly includes eight modules that are initialization and main procedure, internal serial storage read-write procedure, data collection and processing procedures, the interrupt handler, the timer handler, HS12864 display and control program, DA output and bypass relay control program, system self-detection and software anti-interference. Resource allocation of system interrupt uses INT interrupt to interrupt keyboard scanning, timer T0 used for scheduling $100 \mathrm{~ms}$, T1 schedules 1 second.

\section{B. Design of Program Modules}

The main program module of starter working process is shown in Fig.3, each power-up should be initialized, initialization includes the set of PIC16F916 single chip timer, interrupt and so on, write control words of the serial storage chip X25045 and serial LCD driver chip HS12864. New starter's the initial work of new starter should set the initial value of X25045, includes the starting mode, starting voltage, starting current, starting time, stopping time, etc. Four starting 
way is set in the system, according to the set starting way and starting parameters the computer performs soft start and soft stop control. 16-bit LCD alternately displays the information of start parameters. If power supply fails, the power saver program will be performed, bypass relay blocked quickly, enters into the emergency stop. Other modules flow chart are omitted.

\section{TESTING RESUltS}

Measurement and running test have been made in Zibo Galaxy high-tech development co., Ltd.. In the stop state, press the "set up" key to enter soft start mode and parameters setting state, according to the LCD cues, set start mode, the starting time, starting voltage, rated current, current limiting current, soft stop time, current transformer ratio and other parameters, and store in X25045. After accomplished the parameter setting, automatically enter stopped state. Press the start key, the motor enters the soft start state, the system response under four different start mode as shown in Fig.5. After soft start process is complete, close the bypass relay, motor enters the running state automatically, the monitoring motor runs on overload current, when the motor overload and even stalling, according to the inverse relationship of the overload rate and time, protect the motor from over current. In the running mode, press the soft stop key, motor enters into soft stop state, first connects the thyristor MJSY-QKJL module, zero-voltage disconnects bypass relay, one seconds later, the control voltage of thyristor MJSY-QKJL module falls gradually until the voltage is zero. The $80 \mathrm{~kW}$ three phase motor starts under such situations: no-loading start, light loading start, as well as full load start, four kind of voltage current process response curves corresponding with different starting way are obtained as shown in Fig. 5.

Testing results shows that four soft start mode that is current limiting starting, voltage ramp starting, voltage ramp and current restriction soft starting, kick torque starting, according to the motor load choose starting mode, the motor can run in accordance with the set mode and the optimization of starting current and load torque, the smooth starting process, smaller shock current can be achieved. Generally kicks the torque starting way is choose when the motor has full load to start. In the soft start device, current transformer ratio error is less than $0.5 \%$, starting voltage error is less than $1 \%$, starting current error is less than $1 \%$, starting time error is less than $0.1 \%$, stop time error is less than $0.1 \%$, overall startup parameter error of the starter is less than $1 \%$. Especially for high power motor starting.

\section{CONCLUSIONS}

The article proposed using synchronous sampling electric current value inverse time lag protection feature real-time measurement and control method, both reasonable, convenient provides many kinds of soft start ways and protection features, and may in the good solution the hot memory question under the load unceasingly change situation, because the good electro-

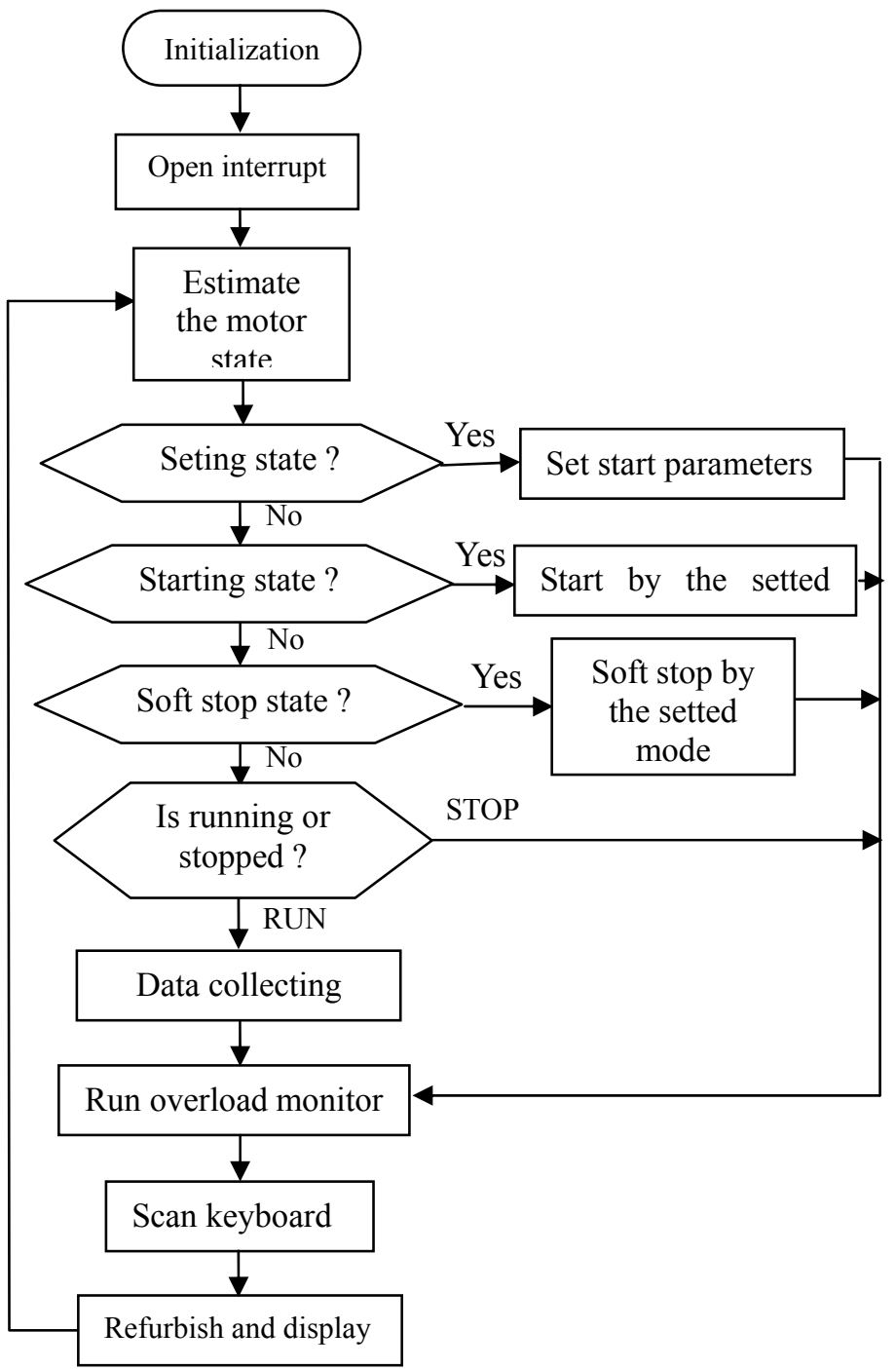

Figure $4 \quad$ Flow chart of main program

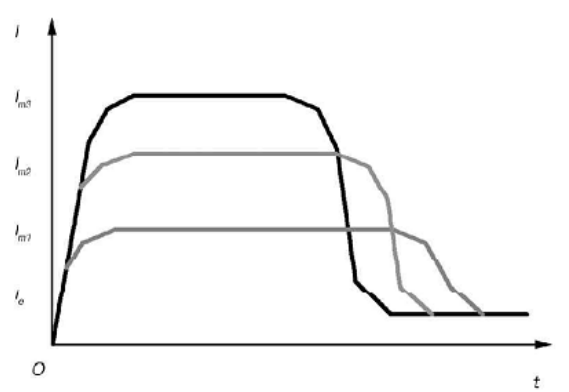

(a) limiting circuit soft start

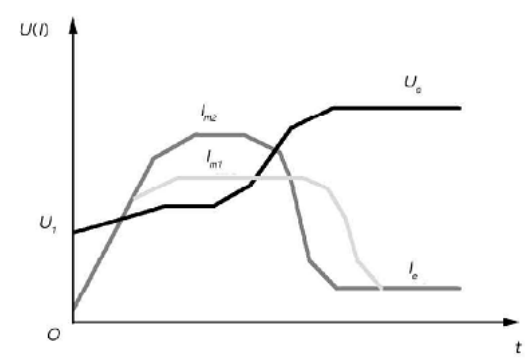

(b) voltage ramp and current restriction soft starting 

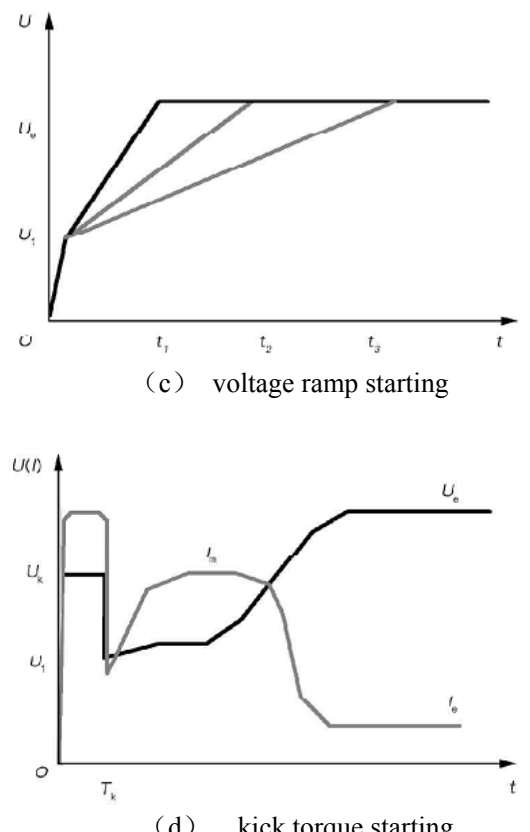

Figure 5 Four kind of voltage current process response curves

optical isolation technology has been used, but also is helpful in enhancing the anti-jamming ability of long timing control unit module. According to the motor load, intelligent soft starter selects one of the four soft start mode. The intelligent soft start carries the situation according to the motor's belting load to carry on four kind of soft start mode selection. The starter produced by Zibo Galaxy high-technology development Limited company indicated that the design technique is novel, the parameter establishment is flexible, the soft start effect is good, the application prospect is broad.

Innovations of the article: adopts thyristor MJSYQKJL module can obtain high control precision; serial chip EEPROM storage flexible and reliable; HS12864 LCD display intuitive, using RS485 communication flexible, current detection and current limiting protection accurate, phase lack protection precisely. Using the I2C bus architecture design for soft starter is more rational, with a more reasonable price.

\section{REFERENCES}

[1] Liu Jinqi,Wang Tao,Xu Wanping,et al.A study on saving energy technology with regulating voltage of induction motor during light loads[C].The $4^{\text {th }}$ International Power Electronics and Motion Control Conference, 2004:409-413.

[2] Zenginobuz G,Cadirci I,Ermis M,et al.Performance optimization of induction motors during voltage-controlled soft starting[J].IEEE Transaction on Energy Conversion,2004,19 (2) : 278-288.

[3] Liu Jinqi,Wang Tao,Xu Wanping,et al.A study on saving energy technology with regulating voltage of induction motor during light loads[C].The $4^{\text {th }}$ International Power Electronics and Motion Control Conference, 2004:409-413.

[4] Xu Dianguo,Zhao Kaiqi,WangYi.A new approach to speed detection and power factor angle control on dsp- based soft-startor-fed IM drive system[C].The 29th Annual Conference of the IEEE,2003: 720-725.

[5] Xu Dianguo,Zhao Kaiqi,Wangyi.Novel method of speed detection and power factor control in thyristor controlled induction motors[C].IEEE International Symposium on Industrial Electronics, 2003: 228-233.

[6] CUI Naxin,ZHANG Chenghui,SUN Fengtao.Study on efficiency optimization and high response control of induction motor[J]. Proceedings of the CSEE, 2005, 25(11): 118-123

Liang Zhang (1987-), male, studys in School of Electric and Electronic Engineering, Shandong University of Technology now, as a graduate student. The major field of study is the power system and automation, which associates with computer intelligent control. E-mail: zhangdaozheng333@126.com

Jishun Jiang(1963-), male, Associate Professor, Master, works in School of Electric and Electronic Engineering, Shandong University of Technology now. The research direction is the computer detection and control theory. E-mail: jandj8@sina.com

Lina Liu(1981-), famale, lecturer, works in School of Electric and Electronic Engineering, Shandong University of Technology now. The research direction is pattern recognition, intelligent control. E-mail: Linaliu-126@163.com 